\title{
The influence of nutritional state on the fatty acid composition of circulating lipid fractions: implications for their use as biomarkers of dietary fat intake
}

\author{
Sion A. Parry ${ }^{a \sharp}$, Fredrik Rosqvist ${ }^{\mathrm{a}, \mathrm{b} \#}$, Sarah Peters ${ }^{\mathrm{a}}$, Rebecca K. Young ${ }^{\mathrm{a}}$, Thomas Cornfield ${ }^{\mathrm{a}}$, Pamela Dyson ${ }^{\mathrm{a}, \mathrm{c}}$ and Leanne \\ Hodson ${ }^{\mathrm{a}, \mathrm{*} *}$ \\ a Oxford Centre for Diabetes, Endocrinology and Metabolism, University of Oxford, Churchill Hospital, Oxford, United Kingdom; ${ }^{\text {b} D e p a r t-~}$

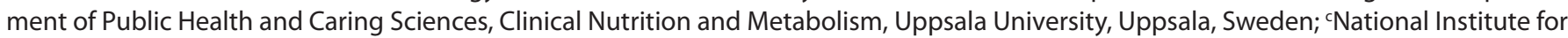 \\ Health Research Oxford, Biomedical Research Centre, Oxford University, Hospital Trusts, Oxford, United Kingdom
}

\begin{abstract}
Background: The fatty acid (FA) composition of blood can be used as an objective biomarker of dietary FA intake. It remains unclear how the nutritional state influences the FA composition of plasma lipid fractions, and thus their usefulness as biomarkers in a non-fasted state.

Objectives: To investigate the associations between palmitate, oleate and linoleate in plasma lipid fractions and self-reported dietary FA intake, and assess the influence of meal consumption on the relative abundance of these FA in plasma lipid fractions (i.e. triglyceride [TG], phospholipids [PLs] and cholesterol esters [CEs]).

Design: Analysis was performed in plasma samples collected from 49 (34 males and 15 females) participants aged 26-57 years with a body mass index (BMI) between 21.6 and $34.2 \mathrm{~kg} / \mathrm{m}^{2}$, all of whom had participated in multiple study visits, thus a pooled cohort of 98 data sets was available for analysis. A subset $(n=25)$ had undergone nutritional interventions and was therefore used to investigate the relationship between the FA composition of plasma lipid fractions and dietary fat intake.

Results: Significant $(P<0.05)$ positive associations were observed between dietary polyunsaturated fat and linoleate abundance in plasma CE. When investigating the influence of meal consumption on postprandial FA composition, we found plasma TG palmitate significantly $(P<0.05)$ decreased across the postprandial period, whereas oleate and linoleate increased. A similar pattern was observed in plasma $\mathrm{PL}$, whereas linoleate abundance decreased in the plasma CE.

Conclusion: Our data demonstrate that the FA composition of plasma CE may be the lipid fraction to utilise as an objective biomarker when investigating recent (i.e. previous weeks-months) dietary FA intakes. In addition, we show that the consumption of a high-fat meal influences the FA composition of plasma TG, $\mathrm{PL}$ and CE over the course of the postprandial period, and therefore, suggest that fasting blood samples should be utilised when using FA composition as a biomarker of dietary FA intake.
\end{abstract}

ARTICLE HISTORY

Received: 17 February 2021

Revised: 16 April 2021

Accepted: 11 May 2021

Published: 13 July 2021

\section{KEYWORDS}

Postprandial; fatty acids; biomarker; lipid fractions; fatty acid composition

\section{Introduction}

The high prevalence of metabolic diseases such as cardiovascular disease (CVD) and type 2 diabetes (T2D) are recognised as a global health issue (1). The relationship between dietary fat quantity and quality and metabolic health is highly debated with some suggesting that increased intake of saturated fat is associated with an increased risk for the development of cardiometabolic diseases (e.g. CVD and T2D), whereas others suggest that no relationship exists (2-5). The conflicting findings may be partly attributable to the methods of dietary assessment used in epidemiological studies, which typically involve the use of food-frequency questionnaires or food diaries, which have known limitations (6-8). Using the fatty acid (FA) composition of blood and tissues as a biomarker of dietary FA intake is an additional and objective method of dietary assessment $(9,10)$. Typically, the FA composition of lipid fractions in blood is measured in the fasting state to avoid the potentially confounding influence of recent dietary FA intake $(11,12)$. However, although it is often assumed that non-fasting samples cannot be used to investigate biomarkers of dietary FA intake, this assumption has not been fully investigated; it remains unclear as to whether nutritional state influences the FA composition of circulating lipid fractions. For large-scale

\#SAP and FR contributed equally to this work.

CONTACT Leanne Hodson leanne.hodson@ocdem.ox.ac.uk

(1) Supplemental data for this article can be accessed here.

(c) 2021 The Author(s). Published by Upsala Medical Society.

This is an Open Access article distributed under the terms of the Creative Commons Attribution License (http://creativecommons.org/licenses/by/4.0/), which permits unrestricted use, distribution, and reproduction in any medium, provided the original work is properly cited. 
observational studies, where it can be logistically challenging to obtain fasting samples, it would be useful to determine whether nutritional state influences circulating FA composition as it may potentially reduce the burden on researchers and participants. Although a number of observational studies have previously obtained non-fasted samples from participants (13-17), or have obtained samples after only a relatively short fasting period (minimum of $4 \mathrm{~h}$ fasting) $(18,19)$, it remains unclear whether the presence of recently ingested fat influenced their findings. Therefore, the aim of this study was to investigate: (1) the association between the dietary saturated (SFA), monounsaturated (MUFA) and polyunsaturated (PUFA) FAs in plasma lipid fractions and self-reported dietary FA intakes, and (2) the influence of meal consumption on the relative abundance of specific SFA, MUFA and PUFA in plasma lipid fractions across a 6 -h postprandial period.

\section{Materials and methods}

\section{Participants}

Participants were recruited from the Oxford Biobank (www. oxfordbiobank.org.uk) (20) or from the wider Oxfordshire area through advertisement. Based on data provided at screening, all volunteers were non-diabetic and free from any known disease, were not taking medication known to affect lipid or glucose metabolism, and did not consume alcohol above recommended limits. Some, but not all, of the data reported in this work constitute a reanalysis of previously published studies $(21,22)$ and ongoing dietary intervention trials (ClinicalTrials.gov identifiers: NCT03090347 and NCT03587753). Data in this manuscript were from a total of 49 (34 males and 15 females) participants aged 26-57 years with a body mass index (BMI) between 21.6 and $34.2 \mathrm{~kg} / \mathrm{m}^{2}$ (Table 1). Twenty-five participants were enrolled in one of two dietary intervention studies, both of which involved changing their relative intakes of fat and carbohydrate. The 25 participants were representative of the study population, as they were aged 38-54 years, with a BMI between 22.0 and $34.2 \mathrm{~kg} / \mathrm{m}^{2}$. Data from this subset were used to investigate the relationship between dietary FA intake and the fasting FA composition of circulating plasma lipid fractions. However, within this subset, four food diaries were missing or incomplete, leaving 46 complete sets of data for analysis.

Table 1. Baseline characteristics of participants.

\begin{tabular}{lc}
\hline Age (years) & $46 \pm 7$ \\
Sex (M/F) & $34 / 15$ \\
BMI (kg/m²) & $26.6 \pm 3.1$ \\
Glucose (mmol/L) & $5.0 \pm 0.6$ \\
Insulin (mU/L) & $8.3 \pm 4.5$ \\
HOMA-IR & $1.9 \pm 1.1$ \\
Total cholesterol (mmol/L) & $4.3 \pm 0.7$ \\
HDL cholesterol (mmol/L) & $1.2 \pm 0.4$ \\
Triglycerides (TGs) (mmol/L) & $0.9 \pm 0.4$ \\
Non-esterified fatty acid ( $\mu$ mol/L) & $519 \pm 275$ \\
\hline Data are mean \pm SD. $n=49$. & \\
M, male; F, female; HDL, high-density lipoprotein; HOMA-IR, Homeostatic \\
Model Assessment for Insulin Resistance.
\end{tabular}

Of the total 49 participants, the remaining 24 participants were enrolled in a randomised crossover study, involving two postprandial study days separated by a 2-week washout period, during which they were asked to maintain their habitual diet and physical activity patterns (21). As all participants $(n=49)$ included in this study took part in two postprandial study visits, this gave a total of 98 data sets to investigate the temporal changes in plasma FA composition in response to meal consumption. A flow chart of participant recruitment and experimental procedures is presented in Supplementary Fig. 1.

All studies were approved by the respective Research Ethics Committee, and all subjects provided written informed consent. Prior to study days, subjects were asked to refrain from strenuous physical activity and to not consume alcohol for a minimum of 24 h. After an overnight fast, subjects came to the Clinical Research Unit at the Oxford Centre for Diabetes, Endocrinology and Metabolism, and a fasting venous blood sample was taken. Subjects were then given a standardised test meal, and venous blood samples were taken at regular intervals for $6 \mathrm{~h}$ after meal consumption.

\section{Dietary interventions and assessments}

A subset $(n=25)$ of participants included in this study underwent dietary interventions. Of these, 16 participated in a randomised crossover trial in which they underwent two dietary interventions: 1) a 4-week low-fat, high-carbohydrate diet enriched in free-sugars and 2) a 4-week high-fat, lowcarbohydrate diet enriched in SFA (22). The remaining nine participants were included in an ongoing study (NCT03090347) and underwent either a 2-week low-fat, high-carbohydrate diet enriched in free-sugars $(n=8)$ or a 2-week high-fat, lowcarbohydrate diet enriched in SFA ( $n=1$ ) (Supplementary Fig. 1).

Dietary intakes were assessed by food diaries collected on 3 days, including a weekend day. Participants taking part in dietary interventions were instructed to maintain their usual body weight, physical activity levels and alcohol intakes throughout the intervention periods, and were contacted weekly by a member of the research team to aid adherence. Dietary intakes were analysed using the Nutritics dietary analysis online software (Dublin, UK), by a registered dietitian to determine energy and nutrient intakes.

\section{Standardised test meal}

On the study day, participants consumed a standardised test meal consisting of $40 \mathrm{~g}$ cereal (Kellogg's Rice Krispies), $200 \mathrm{~g}$ skimmed milk and a chocolate drink containing $40 \mathrm{~g}$ oil, providing $591 \mathrm{kcal}$, with $\sim 64 \%$ energy as fat, $30 \%$ energy as carbohydrate and $\sim 6 \%$ energy as protein. The oil used was either olive oil (Meal A) or $15 \mathrm{~g}$ sunflower oil plus $25 \mathrm{~g}$ palm oil (Meal B). The FA composition of Meal A was $~ 13 \%$ palmitate, $~ 64 \%$ oleate, $\sim 11 \%$ linoleate, $\sim 3 \%$ stearate and $\sim 9 \%$ minor FA, whilst the FA composition of Meal B was $\sim 32 \%$ palmitate, $\sim 35 \%$ oleate, $\sim 27 \%$ linoeate, $\sim 5 \%$ stearate and $\sim 1 \%$ minor FA. All subjects consumed the same meal twice, separated by a period of 2-11 weeks. 

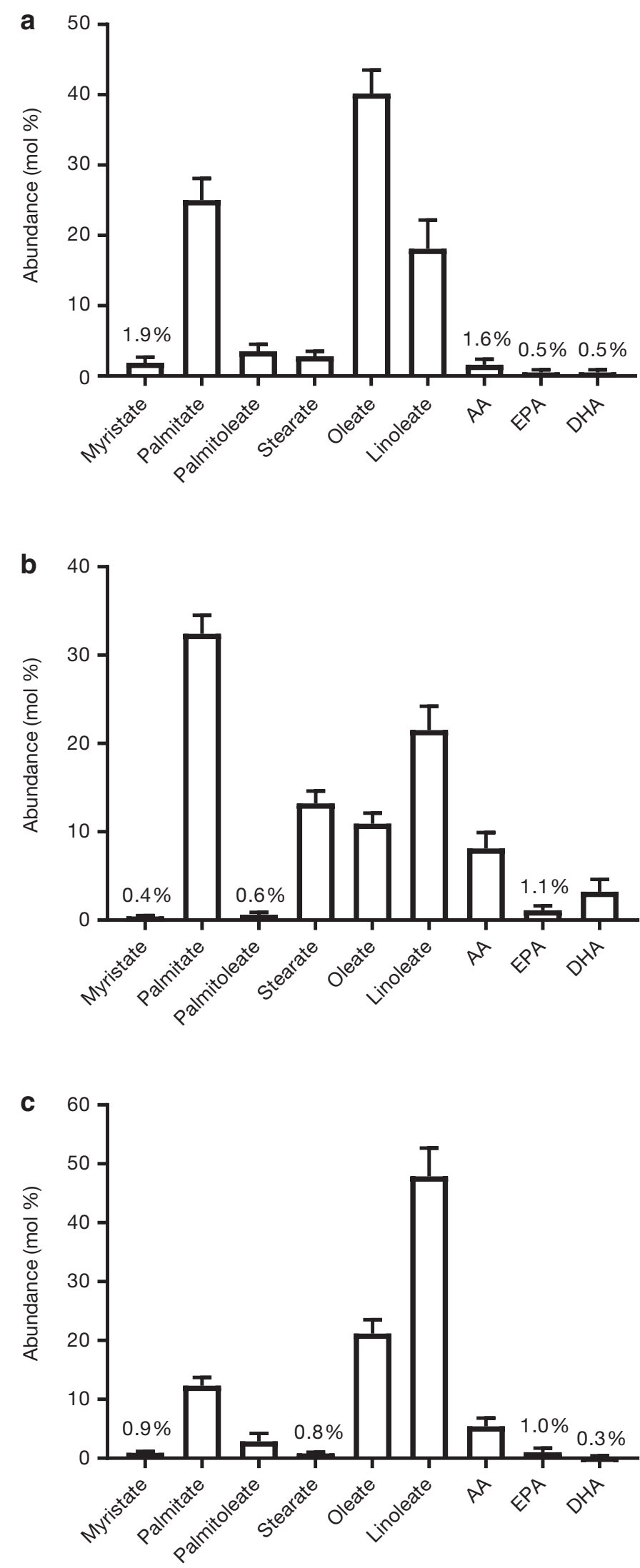

Figure 1. Fasting plasma FA composition for (a) TG $(n=98)$, (b) PL $(n=76)$ and (c) CE $(n=96)$. AA, arachidonic acid; EPA, eicosapentaenoic acid; DHA, docosahexaenoic acid. Data are mean \pm SD.

\section{Analytical procedures}

Whole-blood was collected into heparinised tubes, and plasma was immediately separated for analysis by centrifugation at $4^{\circ} \mathrm{C}$. Plasma glucose, triglycerides (TGs), total cholesterol and highdensity lipoprotein (HDL) cholesterol were analysed enzymatically (llab 600/650 Clinical Chemistry, Werfen).

\section{Fatty acid composition}

Total lipids were extracted from plasma by using chloroformmethanol (2:1, v/v) (23), and plasma lipid fractions (TG, phospholipid [PL] and cholesterol esters [CEs]) were separated by solid-phase extraction (24), followed by methylation using acidified methanol. The FA profile of extracted samples was then determined via gas chromatography with flame ionisation detection (25). FAs were identified by comparing sample retention times to a known standard, and results are expressed as mol\%.

\section{Statistical analysis}

Data were analysed using SPSS (version 25.0). The specific FAs investigated were restricted to the major dietary SFA, MUFA and PUFA (i.e. palmitate, oleate and linoleate, respectively). Normality of variables was assessed by Shapiro-Wilk test and visually by histograms. The Spearmans rank correlation coefficient was used to assess the associations between the specific FAs in lipid fractions and the relative percentages of energy intake from SFA, MUFA and PUFA calculated from 3-day diet diaries. Differences in FA abundance in response to meal consumption were analysed using one-way repeated measures (time) ANOVA. Where a significant main effect of time was noted, Bonferroni post-hoc comparisons were made for postprandial vs. fasting time points (0 min). Data are presented as mean and standard deviation (SD).

\section{Results}

\section{Relationship between dietary FA intake and the FA composition of circulating plasma lipid fractions}

The fasting FA composition of plasma TG, PL and CE fractions is presented in Figure 1. As data were obtained from participants undertaking various dietary interventions, some of which involved increasing dietary carbohydrates/free sugars, and some increasing dietary fat/saturated fat, there were wide variations in self-reported intakes of total fat, SFA, MUFA and PUFA, which is reflective of the specific dietary interventions which were undertaken (Table 2). We assessed the association between dietary FA intake and the abundance of specific FA that represent the major dietary SFA, MUFA and PUFA sources, in the respective plasma lipid fractions. We found no significant associations between dietary FA and the abundance of palmitate, oleate and linoleate in plasma TG or PL (Table 3). However, significant $(P<$ $0.05)$ positive associations were observed between dietary PUFA and linoleate in plasma $C E$, whilst there were no associations between dietary SFA and the abundance of plasma CE palmitate, or dietary MUFA and plasma CE oleate (Table 3). 
Table 2. Dietary fat intake as a proportion of total energy (TE) intake.

\begin{tabular}{lc}
\hline Total fat (\%TE) & $31.5 \pm 13.7$ \\
Saturated fat (\%TE) & $12.3 \pm 7.0$ \\
Polyunsaturated fat (\%TE) & $4.7 \pm 2.5$ \\
Monounsaturated fat (\%TE) & $10.5 \pm 5$ \\
\hline
\end{tabular}

Data are mean \pm SD. $n=46$.

Table 3. Spearmans rank correlation coefficients between the abundance of palmitate, oleate, and linoleate in circulating lipid fractions and the relative percentages of energy intake from dietary saturated fatty acids (SFA), polyunsaturated fatty acids (PUFA) and monounsaturated fatty acids (MUFA).

\begin{tabular}{lccc}
\hline Mol (\%) & $\begin{array}{c}\text { Dietary } \\
\text { SFA (\%TE) }\end{array}$ & $\begin{array}{c}\text { Dietary } \\
\text { MUFA (\%TE) }\end{array}$ & $\begin{array}{c}\text { Dietary } \\
\text { PUFA (\%TE) }\end{array}$ \\
\hline Triglyceride (TG) palmitate & -0.029 & & \\
Phospholipid (PL) palmitate & -0.003 & & \\
Cholesterol ester (CE) palmitate & 0.031 & & \\
TG oleate & & -0.057 & \\
PL oleate & & -0.364 & \\
CE oleate & -0.210 & \\
TG linoleate & & 0.211 \\
PL linoleate & & & 0.198 \\
CE linoleate & & & $0.372^{*}$ \\
\hline
\end{tabular}

*P< 0.05. $n=46$ for TG, $n=27$ for PL and $n=44$ for CE.

\section{Temporal changes in FA composition following meal consumption}

As FA composition is typically measured in the fasting state, we investigated whether the consumption of a meal influences the relative abundance of palmitate, oleate and linoleate in circulating lipid fractions. We achieved this by feeding participants a high-fat test meal, of known FA composition, and assessing changes in plasma palmitate, oleate and linoleate over the course of the postprandial period in the respective lipid fractions.

The consumption of the test meal significantly $(P<0.05)$ decreased the abundance of palmitate in plasma TG, with time points $240-360$ min being significantly $(P<0.05)$ lower than time 0 (fasting), and the greatest differences (i.e. $\sim 1.3 \mathrm{~mol} \%$ ) being apparent between $240 \mathrm{~min}$ and $0 \mathrm{~min}$ (Figure 2a). Conversely, meal consumption significantly $(P<0.05)$ increased the abundance of oleate, and linoleate in plasma TG, with the oleate peaking at $240 \mathrm{~min}$, which was $\sim 3.8 \mathrm{~mol} \%$ greater than 0 min (Figure 2b), and linoleate peaking at time point $360 \mathrm{~min}$, which was $\sim 1.1 \mathrm{~mol} \%$ greater than 0 min (Figure 2c).

For plasma $\mathrm{PL}$, the general trend was similar to the changes observed in plasma TG, although not as striking. The relative abundance of palmitate was significantly $(P<0.05)$ decreased by the consumption of the test meal, with significant differences apparent between time points 240-300 min and 0 min, with a nadir at $300 \mathrm{~min}$, which was $\sim 0.7 \mathrm{~mol} \%$ lower than $0 \mathrm{~min}$ (Figure 3a). The relative abundance of oleate was not influenced by meal consumption (Figure $3 \mathrm{~b}$ ). The abundance of linoleate was significantly increased $(P<0.05)$ following meal consumption, with time points 120 min onwards significantly greater than $0 \mathrm{~min}$, and peaking at $300 \mathrm{~min}$ (i.e. $\sim 1.2 \mathrm{~mol} \%$ greater than 0 min) (Figure $3 c$ ).

In contrast to the FA composition of plasma TG and PL, the consumption of the test meal did not influence the relative
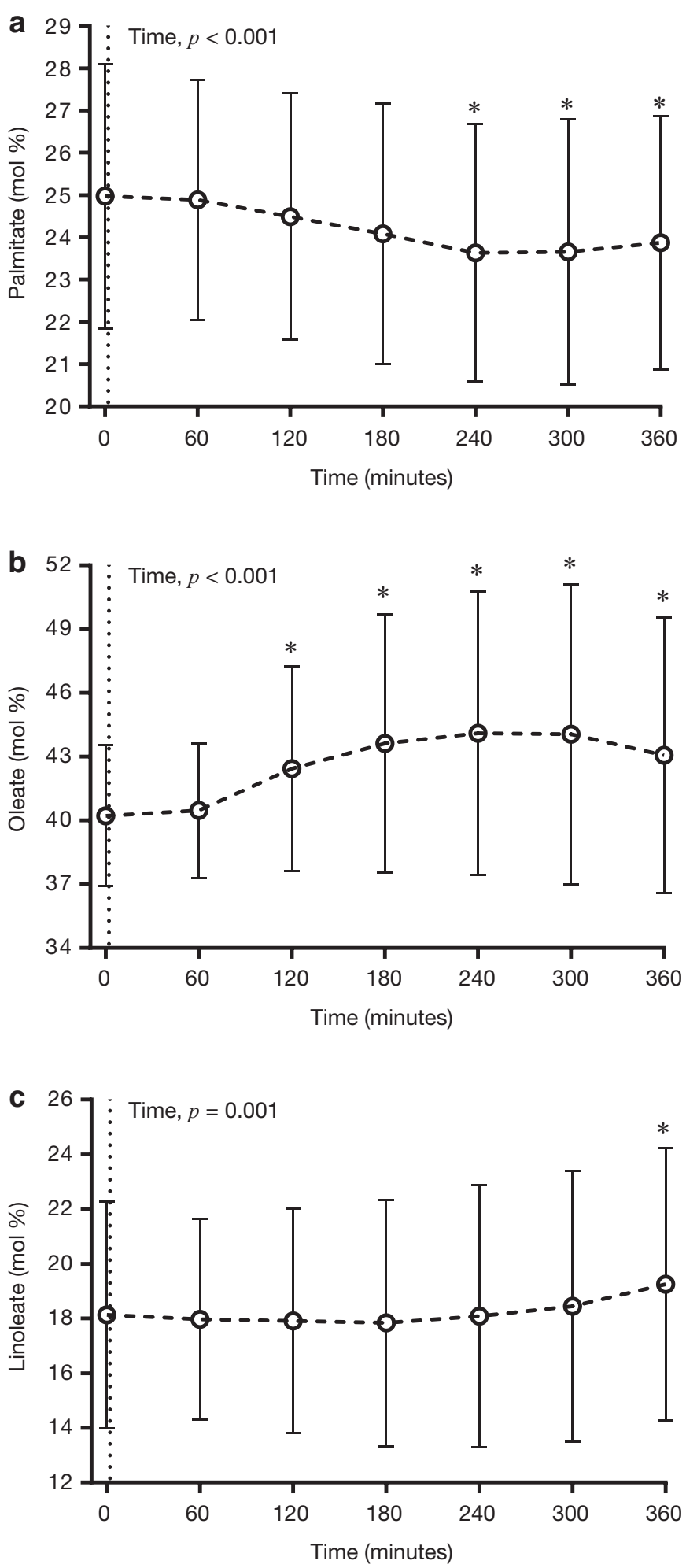

Figure 2. Temporal changes in the relative abundance of (a) palmitate, (b) oleate and (c) linoleate in plasma TG in response to the consumption of a high-fat test meal. Data are presented as mean \pm SD. $n=98$. ${ }^{*} P<0.05 \mathrm{com}$ pared to fasting (Time 0 ). The dotted line at Time 0 denotes the consumption of the experimental test meal.

abundance of palmitate in plasma CE (Figure 4a). There was, however, a significant $(P<0.05)$ main effect of time for oleate in plasma $C E$, although Bonferroni post hoc comparisons revealed no statistically significant differences between any postprandial time 

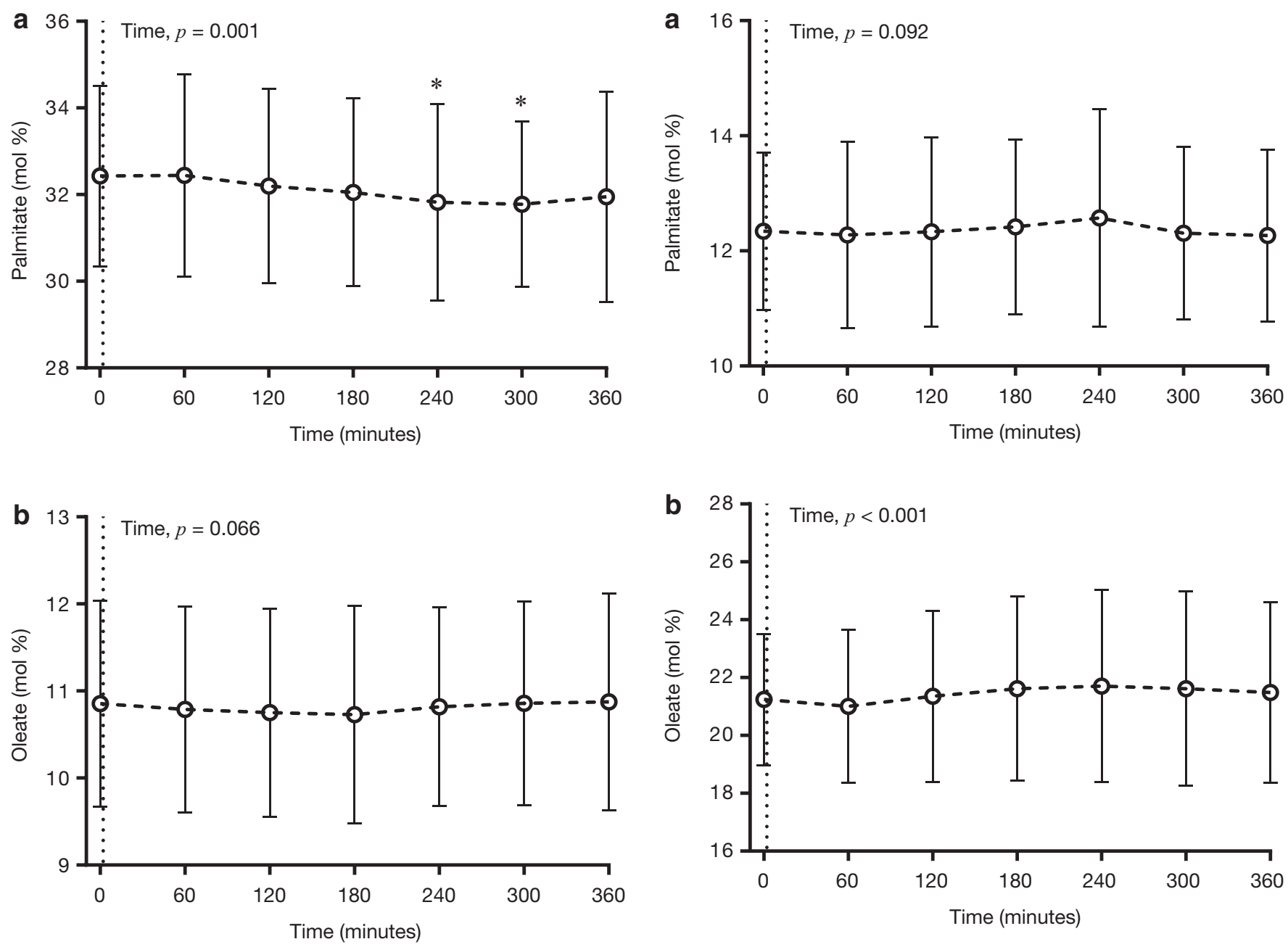

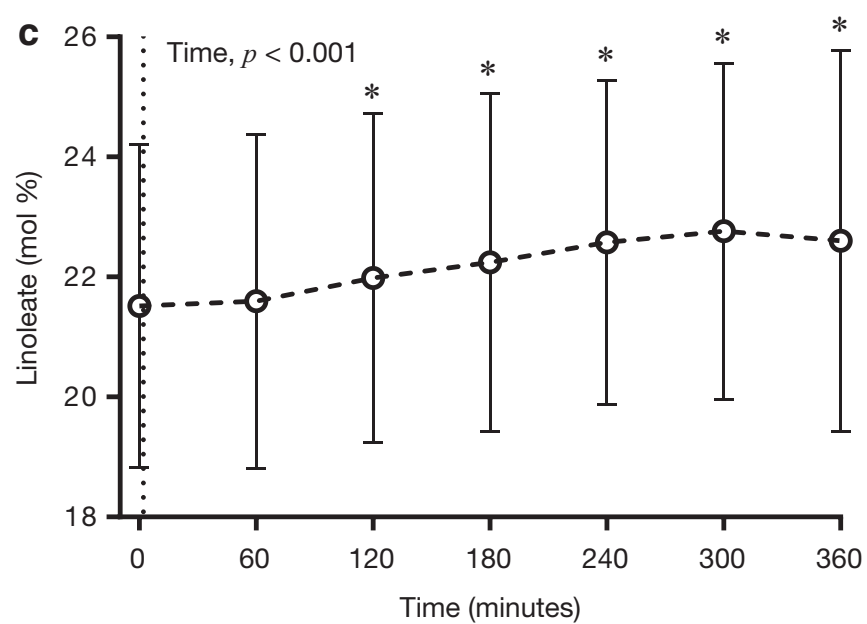

Figure 3. Temporal changes in the relative abundance of (a) palmitate, (b) oleate and (c) linoleate in plasma PL in response to the consumption of a high-fat test meal. Data are presented as mean \pm SD. $n=76$. ${ }^{*} P<0.05$ when compared to fasting (Time 0 ). The dotted line at Time 0 denotes the consumption of the experimental test meal.

points and $0 \mathrm{~min}$ (Figure 4b). The abundance of linoleate in plasma CE significantly $(P<0.05)$ decreased following meal consumption, reaching a nadir at $300 \mathrm{~min}$, which was $\sim 2.6 \mathrm{~mol} \%$ lower than $0 \mathrm{~min}$ (Figure 4).

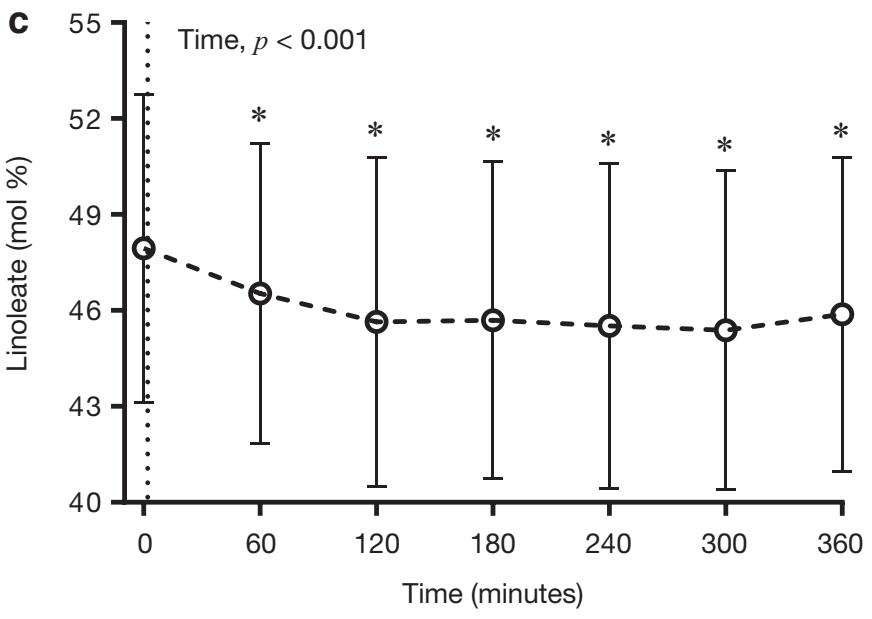

Figure 4. Temporal changes in the relative abundance of (a) palmitate, (b) oleate and (c) linoleate in plasma CE in response to the consumption of a high-fat test meal. Data are presented as mean \pm SD. $n=96$. ${ }^{*} P<0.05$ when compared to fasting (Time 0 ). The dotted line at Time 0 denotes the consumption of the experimental test meal.

\section{Discussion}

The FA composition of blood and tissues has frequently been used as a biomarker of dietary FA intake (9). Typically, the FA composition of blood lipids is measured in blood samples taken 
from individuals after an overnight fast. However, some largescale observational studies have utilised non-fasting samples (13-17), and it remains unclear if feeding influences the postprandial FA composition of plasma lipid fractions. We therefore aimed to assess the associations between FA composition in plasma lipid fractions and dietary FA intake in participants who had undergone dietary interventions to investigate biomarkers of dietary FA intake. In addition, we aimed to determine the influence of meal consumption on the postprandial FA composition of plasma TG, PL and CE. Overall, our findings suggest that the FA composition of plasma TG or PL does not reflect dietary fat intakes over the short term (2-4 weeks), whereas the relative abundance of plasma CE linoleate was positively associated with self-reported intakes of PUFA. Thus, plasma CE linoleate may represent a valid biomarker of dietary PUFA even in situations where individuals have recently altered their dietary FA intakes. Moreover, our data indicate that the consumption of a high-fat test meal acutely influences the FA composition of plasma TG, PL and CE. Therefore, it would seem prudent to suggest that fasting blood samples should be utilised when using FA composition as a biomarker of dietary FA intake, as the consumption of a high-fat meal may confound measurements of FA composition taken in a non-fasting state.

Measurement of FA composition in various tissue and plasma lipid pools provides an objective assessment of dietary FA intake, which may strengthen data obtained from self-reported dietary records which have limitations (e.g. underreporting) (7, $8,26)$. It has been suggested that due to the slow turnover of adipose tissue (i.e. half-life of $\sim 600$ days), adipose tissue FA composition may be most reflective of long-term (i.e. 2-3 years) fat intake (27-29). In contrast, the FA composition of red blood cells, plasma CE and PL has been shown to reflect dietary fat intakes within weeks (29-31). Epidemiological investigations have generally used the FA composition of various plasma/ serum lipid fractions as biomarkers of dietary FA intake $(32,33)$, which may be because blood sampling is relatively simpler than adipose tissue biopsies. However, there is heterogeneity between the metabolism and turnover of circulating lipid fractions, which is reflected in their FA composition, and debate continues as to which plasma lipid fraction represents the most accurate biomarker of dietary fat intake. We therefore investigated the relationship between self-reported dietary fat intake and the relative abundance of palmitate, oleate and linoleate (representative of the major SFA, MUFA and PUFA) in plasma TG, PL and CE.

In line with previous observations (9), we found a positive association between the abundance of linoleate in plasma CE and dietary PUFA intake, but observed no significant associations relative abundance of palmitate or oleate in plasma CE and dietary SFA and MUFA, respectively. We also observed no associations between specific FAs in the plasma TG or PL fraction and self-reported intake of dietary SFA, MUFA and PUFA. It is plausible that positive associations are observed more often for PUFA than other FAs as linoleate represents an essential FA, whereas the in vivo synthesis of palmitate and oleate may influence their circulating abundance independently of dietary intake. Equally, palmitate and oleate are relatively ubiquitous in foods, which, when combined with the inability of FA composition measures to establish quantitative intakes, may make it challenging to separate individuals with low and high intakes of these FA. When comparing the utility of FAs in various lipid fractions as biomarkers of dietary FA intake, Furtado et al. (12) reported that combining plasma TG and non-esterified FA (NEFA) fractions was more reflective of dietary FA intake than total plasma, CE or PL FA composition. The difference between our findings and those of Furtado et al. (12) is likely due to differences in study design. Participants in our study completed 2-4 weeks dietary interventions at the time of assessment, whereas those studied by Furtado et al. (12) had not undertaken a dietary intervention and were consuming their habitual diet, which was assessed using a food frequency questionnaire examining intakes over the previous year. It is therefore plausible that the strength of the correlation for the combined TG and NEFA fraction was driven by NEFA FA composition, which has been suggested to be reflective of adipose tissue FA composition (34).

We found that the abundance of palmitate, oleate and linoleate in plasma TG, PL and CE was influenced by a high-fat meal in a manner which reflected, to a degree, the FA composition of the test meal. Changes in FA abundance were specific to lipid fractions. The plasma TG and PL fractions were influenced to a greater extent than plasma CE. It is unsurprising that the composition of circulating TG was altered during the postprandial period as ingested FAs are initially incorporated into chylomicron-TG prior to entering the circulation (35), and the incorporation of dietary FA into hepatic very low-density lipoprotein (VLDL) TG also occurs relatively soon after ingestion (25). Thus, our data are in-line with others who have previously demonstrated that changes in the non-fasting FA composition of plasma TG are reflective of the recently ingested meal FA composition (36-38). We also show that the FA composition of plasma PL and CE is influenced by the intake of a high-fat mixed meal with significant differences apparent from 60-min onwards, dependent on the fraction and FA. Recently, Shokry et al. demonstrated that the abundance of some FA in plasma TG and NEFA, including linoleate, changed during a 7-h postprandial period following a high-calorie mixed macronutrient test meal (45 $\mathrm{g}$ of fat and $97 \mathrm{~g}$ of carbohydrate); the plasma PL FA composition was unaffected (38). This finding is in contrast to our observations, but may be explained in part by the difference in the FA composition of the meals, with Shokry et al. feeding a test meal containing $26.4 \mathrm{~g}$ of linoleate (i.e. over $50 \%$ of the fat component). Meal consumption has also been shown to influence the FA composition of plasma PL when assessed using lipidomic methodologies $(39,40)$. Our observations are in line with Karupaiah et al. who reported changes in plasma CE within 7-h, which reflected the fat composition of the consumed meal (41). Thus, regardless of methodology (GC or lipidomics), it would seem that changes in both PL and CE species have been observed in the non-fasting compared with the fasting state, but the degree and manner of change may be dependent on the composition of the test meal. 
In the present work, the abundance of linoleate increased in the TG and PL fractions but decreased in the CE. These differences may be explained by differences in FA incorporation time between the fractions, as the synthesis of $C E$ involves the enzymatic transfer of an FA from PL and cholesterol precursors, typically from the sn-2 position of $\mathrm{PL}$, which is commonly occupied by a PUFA (9). Using stable isotope tracer methodology, we have previously shown the incorporation of linoleate in plasma PL is greater than palmitate following a high-fat mixed meal (21), demonstrating the metabolic heterogeneity of specific FA in lipid fractions. It is therefore plausible that the incorporation time of linoleate in plasma CE from $\mathrm{PL}$ is longer than we have investigated, and that the abundance of linoleate in plasma CE may have increased at a time point later than the 6-h period examined here.

Our study has a number of limitations, including: all subjects were free from known metabolic disease, and results may therefore not be reflective of other metabolic phenotypes. For instance, individuals with metabolic-associated fatty liver disease (MAFLD) demonstrate increased de novo lipogenesis (DNL) relative to their non-MAFLD counterparts (42), which may lead to an increased palmitate abundance in plasma lipid fractions. Participants consumed a single test meal, which was high in fat; therefore, we cannot exclude the possibility that non-fasting FA composition would change further if we had given a subsequent/second meal (i.e. more reflective of habitual dietary pattern in most individuals). Moreover, it remains unclear if the responses observed were mediated by the quantity, along with the quality of FA in the meal; it could be speculated that a lower fat meal may result in less notable/obvious responses/ changes. Our test meals were devoid of marine n-3 FA; thus, we are unable to comment on the stability of these FA across the postprandial period, but it would be of interest to investigate this given their usefulness as biomarkers (43-45). Similarly, we only assessed the most abundant SFA, MUFA and PUFA; therefore, our findings cannot be extrapolated to FA of lower abundance, for example, myristate, pentadecanoic acid, stearate and arachidonic acid. We did not assess the FA composition of plasma NEFA, which whilst being a potentially good marker of long-term dietary FA intake (as it reflects AT FA composition) (9), likely does not reflect short- to medium-term dietary intake. In addition, we and others have previously shown that during the postprandial period chylomicron-derived dietary FA spillover contributes $10-50 \%$ of FA within the systemic NEFA pool (46-48). Thus, similar to plasma TG FA composition with meal consumption, the FA composition of plasma NEFA would be highly influenced over the postprandial period by the fat content and FA composition of the recently consumed meal.

In conclusion, our data demonstrate that the FA composition of plasma TG and CE does not reflect short-term (i.e. previous weeks) dietary FA intakes, and that the FA composition of plasma CE may be the lipid fraction to utilise as an objective biomarker when investigating recent dietary FA intakes over this period. In addition, we show that the consumption of a high-fat meal influences the FA composition of plasma TG, PL and CE over the course of the postprandial period, with responses appearing to be specific to FAs in the different lipid fractions. Thus, the FA composition of plasma lipid fractions during the postprandial period (i.e. 1-6 h post meal) may not reflect fasting values. Based on these observations, it would be prudent to suggest that fasting blood samples should be utilised when using FA composition as a biomarker of dietary FA intake.

\section{Acknowledgements}

We thank Louise Dennis, Rachel Craven-Todd and all CRU staff for excellent nursing provision, and Niall Dempster and Lia Anguelova (Oxford Centre for Diabetes, Endocrinology and Metabolism, University of Oxford, Oxford) for providing medical support during study days. This work was supported by the NIHR Biomedical Research Centre, Oxford, and we also thank the volunteers from the Oxford Biobank, NIHR Oxford Biomedical Research Centre, for their participation. The Oxford Biobank (www.oxfordbiobank.org.uk) is also a part of the NIHR National Bioresource, which supported the recalling process of the volunteers.

\section{Conflict of interest}

All authors declare no conflict of interest.

\section{Disclosure statement}

The authors have no conflicts of interest to declare.

\section{Funding}

$\mathrm{LH}$ is a British Heart Foundation Senior Research Fellow in Basic Science (FS/15/56/31645). Work contributing to this manuscript was funded by the World Sugar Research Organization, and the Biotechnology and Biological Sciences Research Council UK (BB/ N005600/1 and BB/N015665/1). During this work, FR was supported by the Henning and Johan Throne-Holsts Foundation, Swedish Society for Medical Research, Swedish Society of Medicine and The Foundation Blanceflor.

\section{Notes on contributors}

Sion A. Parry, Sion A. Parry, PhD is a researcher at the Oxford Centre for Diabetes, Endocrinology and Metabolism (OCDEM), University of Oxford, United Kingdom.

Fredrik Rosqvist, Fredrik Rosqvist, PhD was a postdoctoral researcher at the Oxford Centre for Diabetes, Endocrinology and Metabolism (OCDEM), University of Oxford, United Kingdom at the time of the work, now a researcher at the Department of public health and caring sciences, Clinical nutrition and metabolism, Uppsala University, Sweden.

Sarah Peters, Sarah Peters was an undergraduate medical student undertaking a final honours scheme research project at the Oxford Centre for Diabetes, Endocrinology and Metabolism 
(OCDEM), University of Oxford, United Kingdom at the time of the work.

Rebecca Young, Rebecca Young was an undergraduate medical student was an undergraduate medical student undertaking a final honours scheme research project at the Oxford Centre for Diabetes, Endocrinology and Metabolism (OCDEM), University of Oxford, United Kingdom at the time of the work.

Thomas Cornfield, Thomas Cornfield, BSc was a research technician at the Oxford Centre for Diabetes, Endocrinology and Metabolism (OCDEM), University of Oxford, United Kingdom at the time of the work.

Pamela Dyson, Pamela Dyson, $\mathrm{PhD}$ is a research dietitian at the Oxford Centre for Diabetes, Endocrinology and Metabolism (OCDEM), University of Oxford, United Kingdom.

Leanne Hodson, Leanne Hodson, $\mathrm{PhD}$ is a Professor of Metabolic Physiology and British Heart Foundation Senior Research Fellow at the Oxford Centre for Diabetes, Endocrinology and Metabolism (OCDEM), University of Oxford, United Kingdom.

\section{ORCID}

Sion A Parry (D) https://orcid.org/0000-0002-9037-1619

Fredrik Rosqvist (D) https://orcid.org/0000-0002-8982-6129

Sarah Peters (D) https://orcid.org/0000-0003-0094-8638

Pamela Dyson (D) https://orcid.org/0000-0001-5391-6301

Leanne Hodson (D) https://orcid.org/0000-0002-2648-6526

\section{References}

1. Ralston J, Nugent R. Toward a broader response to cardiometabolic disease. Nat Med. 2019;25:1644-6. doi: 10.1038/s41591-019-0642-9

2. Astrup A, Magkos F, Bier DM, Brenna JT, de Oliveira Otto MC, Hill JO, et al. Saturated Fats and Health: A Reassessment and Proposal for FoodBased Recommendations: JACC State-of-the-Art Review. J Am Coll Cardiol. 2020;76:844-57. doi: 10.1016/j.jacc.2020.05.077

3. Chowdhury R, Warnakula S, Kunutsor S, Crowe F, Ward HA, Johnson L, et al. Association of dietary, circulating, and supplement fatty acids with coronary risk: a systematic review and meta-analysis. Ann Intern Med. 2014;160:398-406. doi: 10.7326/M13-1788

4. Jakobsen MU, O'Reilly EJ, Heitmann BL, Pereira MA, Balter K, Fraser GE, et al. Major types of dietary fat and risk of coronary heart disease: a pooled analysis of 11 cohort studies. Am J Clin Nutr. 2009;89:1425-32. doi: 10.3945/ajcn.2008.27124

5. Willett WC. Dietary fats and coronary heart disease. J Intern Med. 2012;272:13-24. doi: 10.1111/j.1365-2796.2012.02553.x

6. SShim JS, Oh K, Kim HC. Dietary assessment methods in epidemiologic studies. Epidemiol Health. 2014;36:e2014009. doi: 10.4178/epih/e2014009

7. Bingham SA. Biomarkers in nutritional epidemiology. Public Health Nutr. 2002;5:821-7. doi: 10.1079/PHN2002368

8. Jenab M, Slimani N, Bictash M, Ferrari P, Bingham SA. Biomarkers in nutritional epidemiology: applications, needs and new horizons. Hum Genet. 2009;125:507-25. doi: 10.1007/s00439-009-0662-5

9. Hodson L, Skeaff CM, Fielding BA. Fatty acid composition of adipose tissue and blood in humans and its use as a biomarker of dietary intake. Prog Lipid Res. 2008;47:348-80. doi: 10.1016/j.plipres.2008.03.003

10. Wolk A, Furuheim M, Vessby B. Fatty acid composition of adipose tissue and serum lipids are valid biological markers of dairy fat intake in men. J Nutr. 2001;131:828-33. doi: 10.1093/jn/131.3.828
11. Marchioni DM, de Oliveira MF, Carioca AAF, Miranda AAM, Carvalho AM, Oki E, et al. Plasma fatty acids: Biomarkers of dietary intake? Nutrition. 2019;59:77-82. doi: 10.1016/j.nut.2018.08.008

12. Furtado JD, Beqari J, Campos H. Comparison of the Utility of Total Plasma Fatty Acids Versus those in Cholesteryl Ester, Phospholipid, and Triglyceride as Biomarkers of Fatty Acid Intake. Nutrients. 2019;11. doi: 10.3390/nu11092081

13. Clarke R, Shipley M, Armitage J, Collins R, Harris W. Plasma phospholipid fatty acids and CHD in older men: Whitehall study of London civil servants. Br J Nutr. 2009;102:279-84. doi: 10.1017/S0007114508143562

14. Sun $Q$, Ma J, Campos H, Hankinson SE, Manson JE, Stampfer MJ, et al. A prospective study of trans fatty acids in erythrocytes and risk of coronary heart disease. Circulation. 2007;115:1858-65. doi: 10.1161/ CIRCULATIONAHA.106.679985

15. Sun Q, Ma J, Campos H, Hu FB. Plasma and erythrocyte biomarkers of dairy fat intake and risk of ischemic heart disease. Am J Clin Nutr. 2007;86:929-37. doi: 10.1093/ajcn/86.4.929

16. Sun Q, Ma J, Campos H, Rexrode KM, Albert CM, Mozaffarian D, et al. Blood concentrations of individual long-chain n-3 fatty acids and risk of nonfatal myocardial infarction. Am J Clin Nutr. 2008;88:216-23. doi: 10.1093/ajcn/88.1.216

17. Warensjo E, Jansson JH, Cederholm T, Boman K, Eliasson M, Hallmans G, et al. Biomarkers of milk fat and the risk of myocardial infarction in men and women: a prospective, matched case-control study. Am J Clin Nutr. 2010;92:194-202. doi: 10.3945/ajcn.2009.29054

18. Hallgren CG, Hallmans G, Jansson JH, Marklund SL, Huhtasaari F, Schutz A, et al. Markers of high fish intake are associated with decreased risk of a first myocardial infarction. Br J Nutr. 2001;86:397-404. doi: 10.1079/ BJN2001415

19. Warensjo E, Jansson JH, Berglund L, Boman K, Ahren B, Weinehall L, et al. Estimated intake of milk fat is negatively associated with cardiovascular risk factors and does not increase the risk of a first acute myocardial infarction. A prospective case-control study. Br J Nutr. 2004;91:635-42. doi: 10.1079/BJN20041080

20. Karpe F, Vasan SK, Humphreys SM, Miller J, Cheeseman J, Dennis AL, et al. Cohort Profile: The Oxford Biobank. Int J Epidemiol. 2018;47:21-g doi: $10.1093 /$ ije/dyx132

21. Parry SA, Rosqvist F, Cornfield T, Barrett A, Hodson L. Oxidation of dietary linoleate occurs to a greater extent than dietary palmitate in vivo in humans. Clin Nutr. 2020. doi: 10.1016/j.clnu.2020.07.013

22. Parry SA, Rosqvist F, Mozes FE, Cornfield T, Hutchinson M, Piche ME, et al. Intrahepatic Fat and Postprandial Glycemia Increase After Consumption of a Diet Enriched in Saturated Fat Compared With Free Sugars. Diabetes Care. 2020;43:1134-41. doi: 10.2337/dc19-2331

23. Folch J, Ascoli I, Lees M, Meath JA, Le BN. Preparation of lipide extracts from brain tissue. J Biol Chem. 1951;191:833-41. doi: 10.1016/ S0021-9258(18)55987-1

24. Burdge $G C$, Wright $P$, Jones $A E$, Wootton $S A$. A method for separation of phosphatidylcholine, triacylglycerol, non-esterified fatty acids and cholesterol esters from plasma by solid-phase extraction. Br J Nutr. 2000;84:781-7. doi: 10.1017/S0007114500002154

25. Heath RB, Karpe F, Milne RW, Burdge GC, Wootton SA, Frayn KN. Selective partitioning of dietary fatty acids into the VLDL TG pool in the early postprandial period. J Lipid Res. 2003;44:2065-72. doi: 10.1194/jlr. M300167-JLR200

26. King IB, Lemaitre RN, Kestin M. Effect of a low-fat diet on fatty acid composition in red cells, plasma phospholipids, and cholesterol esters: investigation of a biomarker of total fat intake. Am J Clin Nutr. 2006;83:227-36. doi: 10.1093/ajcn/83.2.227

27. Dayton S, Hashimoto S, Dixon W, Pearce ML. Composition of lipids in human serum and adipose tissue during prolonged feeding of a diet high in unsaturated fat. J Lipid Res. 1966;7:103-11. doi: 10.1016/ S0022-2275(20)39591-2

28. Hirsch J, Farquhar JW, Ahrens EH, Jr., Peterson ML, Stoffel W. Studies of adipose tissue in man. A microtechnic for sampling and analysis. Am J Clin Nutr. 1960;8:499-511. doi: 10.1093/ajcn/8.4.499 
29. Katan MB, Deslypere JP, van Birgelen AP, Penders M, Zegwaard M. Kinetics of the incorporation of dietary fatty acids into serum cholesteryl esters, erythrocyte membranes, and adipose tissue: an 18-month controlled study. J Lipid Res. 1997;38:2012-22. doi: 10.1016/S0022-2275(20)37132-7

30. Hodson L, Eyles HC, McLachlan KJ, Bell ML, Green TJ, Skeaff CM. Plasma and erythrocyte fatty acids reflect intakes of saturated and n-6 PUFA within a similar time frame. J Nutr. 2014;144:33-41. doi: 10.3945/jn.113.183749

31. Skeaff CM, Hodson L, McKenzie JE. Dietary-induced changes in fatty acid composition of human plasma, platelet, and erythrocyte lipids follow a similar time course. J Nutr. 2006;136:565-9. doi: 10.1093/jn/136.3.565

32. Del Gobbo LC, Imamura F, Aslibekyan S, Marklund M, Virtanen JK, Wennberg $M$, et al. omega-3 Polyunsaturated Fatty Acid Biomarkers and Coronary Heart Disease: Pooling Project of 19 Cohort Studies. JAMA Intern Med. 2016;176:1155-66. doi: 10.1001/jamainternmed.2016.2925

33. Marklund M, Wu JHY, Imamura F, Del Gobbo LC, Fretts A, de Goede J, et al. Biomarkers of Dietary Omega-6 Fatty Acids and Incident Cardiovascular Disease and Mortality. Circulation. 2019;139:2422-36. doi: 10.1161/ CIRCULATIONAHA.118.038908

34. Hellmuth C, Demmelmair H, Schmitt I, Peissner W, Bluher M, Koletzko B. Association between plasma nonesterified fatty acids species and adipose tissue fatty acid composition. PLoS One. 2013;8:e74927. doi: 10.1371/journal.pone.0074927

35. Fielding BA, Frayn KN. Lipoprotein lipase and the disposition of dietary fatty acids. Br J Nutr. 1998;80:495-502. doi: 10.1017/S0007114598001585

36. Burdge GC, Powell J, Calder PC. Lack of effect of meal fatty acid composition on postprandial lipid, glucose and insulin responses in men and women aged 50-65 years consuming their habitual diets. Br J Nutr. 2006;96:489-500.

37. Strassburg K, Esser D, Vreeken RJ, Hankemeier T, Muller M, van Duynhoven J, et al. Postprandial fatty acid specific changes in circulating oxylipins in lean and obese men after high-fat challenge tests. Mol Nutr Food Res. 2014;58:591-600. doi: 10.1002/mnfr.201300321

38. Shokry E, Raab R, Kirchberg FF, Hellmuth C, Klingler M, Demmelmair H, et al. Prolonged monitoring of postprandial lipid metabolism after a western meal rich in linoleic acid and carbohydrates. Appl Physiol Nutr Metab. 2019;44:1189-98. doi: 10.1139/apnm-2018-0798

39. Grace MS, Dempsey PC, Sethi P, Mundra PA, Mellett NA, Weir JM, et al. Breaking Up Prolonged Sitting Alters the Postprandial Plasma Lipidomic
Profile of Adults With Type 2 Diabetes. J Clin Endocrinol Metab. 2017;102:1991-9. doi: 10.1210/jc.2016-3926

40. Meikle PJ, Barlow CK, Mellett NA, Mundra PA, Bonham MP, Larsen A, et al. Postprandial Plasma Phospholipids in Men Are Influenced by the Source of Dietary Fat. J Nutr. 2015;145:2012-8. doi: 10.3945/jn.115.210104

41. Karupaiah T, Sundram K. Modulation of human postprandial lipemia by changing ratios of polyunsaturated to saturated $(\mathrm{P} / \mathrm{S})$ fatty acid content of blended dietary fats: a cross-over design with repeated measures. Nutr J. 2013;12:122. doi: 10.1186/1475-2891-12-122

42. Smith Gl, Shankaran M, Yoshino M, Schweitzer GG, Chondronikola M, Beals JW, et al. Insulin resistance drives hepatic de novo lipogenesis in nonalcoholic fatty liver disease. J Clin Invest. 2020;130:1453-60. doi: 10.1172/JCI134165

43. Kuriki K, Nagaya T, Tokudome Y, Imaeda N, Fujiwara N, Sato J, et al. Plasma concentrations of ( $n-3)$ highly unsaturated fatty acids are good biomarkers of relative dietary fatty acid intakes: a cross-sectional study. J Nutr. 2003;133:3643-50. doi: 10.1093/jn/133.11.3643

44. Marklund M, Magnusdottir OK, Rosqvist F, Cloetens L, Landberg R, Kolehmainen $\mathrm{M}$, et al. A dietary biomarker approach captures compliance and cardiometabolic effects of a healthy Nordic diet in individuals with metabolic syndrome. J Nutr. 2014;144:1642-9. doi: 10.3945/ jn.114.193771

45. Uusitupa M, Hermansen K, Savolainen MJ, Schwab U, Kolehmainen M, Brader $L$, et al. Effects of an isocaloric healthy Nordic diet on insulin sensitivity, lipid profile and inflammation markers in metabolic syndrome -- a randomized study (SYSDIET). J Intern Med. 2013;274:52-66. doi: 10.1111/joim.12044

46. Barrows BR, Parks EJ. Contributions of different fatty acid sources to very low-density lipoprotein-triacylglycerol in the fasted and fed states. J Clin Endocrinol Metab. 2006;91:1446-52. doi: 10.1210/ jc.2005-1709

47. Barrows BR, Timlin MT, Parks EJ. Spillover of dietary fatty acids and use of serum nonesterified fatty acids for the synthesis of VLDL-triacylglycero under two different feeding regimens. Diabetes. 2005;54:2668-73. doi: 10.2337/diabetes.54.9.2668

48. Piche ME, Parry SA, Karpe F, Hodson L. Chylomicron-Derived Fatty Acid Spillover in Adipose Tissue: A Signature of Metabolic Health? J Clin Endocrinol Metab. 2018;103:25-34. doi: 10.1210/jc.2017-01517 